\title{
Enhancement for Power Quality in Distribution Side Using Custom Power Devices
}

\author{
Dr.S. Suresh Kumar ${ }^{1 \mathrm{a}}$, M. Kalyanasundaram ${ }^{2 \mathrm{~b}}$, Ammu Wilson ${ }^{3 \mathrm{c}}$ \\ ${ }^{I}$ Dr.S.Suresh Kumar, Vice Chairman, IET, Principal Vivekanandha College of Technology for \\ Women,Tamilnadu \\ ${ }^{2}$ M.Kalyanasundaram,Asst Professor/EEE,Vivekananadha College of Engg for Women, Tamilnadu,India \\ ${ }^{3}$ Ammu Wilson, PG Scholar, Vivekanandha College of Engg for Women,Tamilnadu,India.
}

\begin{abstract}
Power quality (PQ) of power operating device is one of today's most discussed topic but also it is a most problematic subject in recent years. With increasing applications of nonlinear and electronically switched devices in distribution systems and industries, power-quality $(P Q)$ problems, such as harmonics, neutral current elimination, reactive power has become an unavoidable issues. The introduction of Unified Power Quality Conditioner in distribution side in one feeder network has made it possible to mitigate the following problems effectively. Interline Unified Power Quality Conditioner which is installed between two feeders is its one of the advancement .The controlling algorithm determines the production of controlling signals which is used by the Voltage Source Converters for the generation of their gating signals. The variation of performance of the device occurs with the different controlling algorithms. This paper proposes the usage of Synchronous Reference Frame Controlling algorithm for the gate signal generation and the usage of modified PLL for better performance. Due to the effective usage of fuzzy controller based on PSO algorithm in the series side of the device make it more advantageous than other commonly used controllers. The main power quality issues we concentrate upon relates to reactive power compensation, harmonics elimination and neutral current elimination. The output has been plotted in MATLAB/SIMULINK.

Index terms: Interline Unified Power Quality Conditioner(IUPQC),Power Quality (PQ), Phase Locked Loop (PLL),Synchronous Reference Frame (SRF),Active Pass Filter(APF)
\end{abstract}

\section{Introduction}

An electric power system is a network of electrical components used to supply, transmit and use electric power. It is the network that supplies a region's homes and industry with power - for sizable regions, this power system is known as the grid and can be broadly divided into the generators that supply

the power, the transmission system that carries the power from the generating centre's to the load centre's and the distribution system that feeds the power to nearby homes and industries [1].

Power quality determines the fitness of electric power to consumer devices. Synchronization of the voltage frequency and phase allows electrical systems to function in their intended manner without significant loss of performance or life. The term is used to describe electric power that drives an electrical load and the load's ability to function properly. Without the proper power, an electrical device (or load) may malfunction, fail prematurely or not operate at all. There are many ways in which electric power can be of poor quality and many more causes of such poor quality power. Poor power quality may result into increased power losses, and other remarkable abnormalities in the distribution side. The problems became more serious with the high usage of non-linear loads. The main reason for this is that the nonlinear loads, as a rule, draw non sinusoidal currents from the supply and lead to voltage distortion and related system problems [3].

APFs have made it possible to mitigate some of the major power quality problems effectively Power conditioners are typically based on IGBT PWM converters and connected to low and medium voltage distribution systems in shunt, series or both at the same time. Choosing a kind of equipment to improve the power quality depends on the source of the problem.

The Unified Power Quality Conditioner (UPQC) is one of the key custom power device, which can compensate both current and voltage related problems, Simultaneously. The UPQC is connected before the load to make the load voltage free from any distortions and at the same time, the reactive current drawn from source should be compensated in such a way that the currents at source side, would be in phase with utility voltages[4].

\section{System Configuration}

One of the foremost custom power devices that are competent of alleviating the consequence of power quality problems at the non linear load is the UPQC . In addition to removal of harmonics, recompense for reactive power, load current unbalance, source voltage sags, source voltage unbalance and power factor 
correction are provided by UPQCs. Generally, an UPQC is comprised of two voltage source inverters (VSIs) sharing with one DC link capacitor. Here, the main problem is that the discharging time of DC link capacitor is very high. To mitigate this problem, an PID controller with synchronous reference frame controlling algorithm is made. Rather than the conventional PLL. This paper proposes the usage of modified PLL due to its large advantages. The control circuit of UPQC is shown in figure 1 .

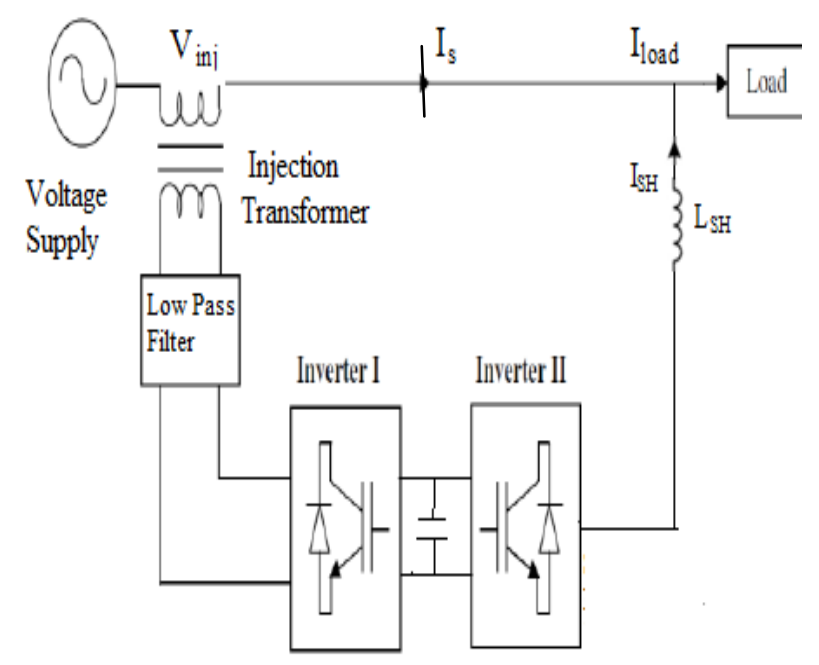

Fig 1 Control circuit of UPQC

\section{S.R.F METHOD}

In the synchronously rotating de - qe reference frame, the components at the fundamental frequency, are transformed to dc quantities and all the harmonics are transformed to non-dc quantities and undergo a frequency shift of $60 \mathrm{~Hz}$ in the spectrum. SRF controller extracts the dc quantities by a low-pass filter (LPF) and hence it is insensitive to phase errors. This is a significant advantage of the SRF controller since most other controllers will introduce significant phase errors at fundamental and at harmonic frequencies. The synchronous reference frame theory mainly uses Phase Lock Loop (PLL) signal, for generation of fundamental frequency unit vectors[5] .

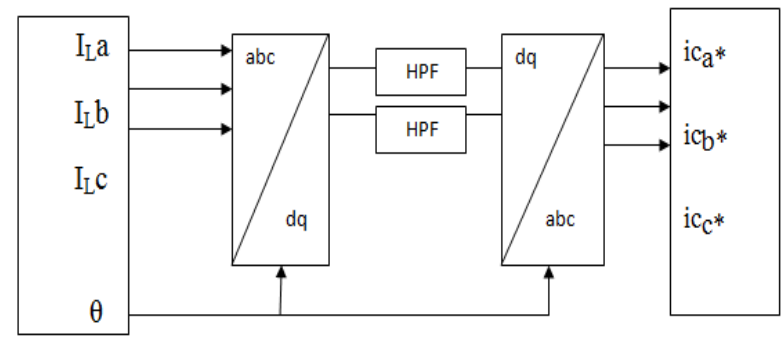

Figure 2: SRF control method

In the SRF method shown in figure $2, \theta$ is a time varying angle that represents the angular position of the reference frame which is rotating at constant speed in synchronism with the three phase ac voltages. To implement the SRF method some kind of synchronizing system should be used. phase-locked loop (PLL) is used for the implementation of this method. In this case the speed of the reference frame is practically constant, that is, the method behaves as if the reference frame's moment of inertia is infinite. The fundamental currents of the $\mathrm{d}-\mathrm{q}$ components are now dc values. The harmonics appear like ripple. Harmonic isolation of the d-q transformed signal is achieved by removing the dc offset. This is accomplished using high pass filters (HPF). In spite of a high pass filter, a low pass filter is used to obtain the reference source current in d-q coordinates. There is no need to supply voltage waveform for a SRF based controller. However the phase position angle must be determined using voltage information. In this paper it mainly uses the modified PLL for its advantage in its filtering performance. For both shunt and series APF SRF with modified PLL is used.

In this paper the synchronous reference frame theory is using a transformation matrix named as $\mathrm{T}$ and is given as equation 1 


$$
\sqrt{2 / 3}\left[\begin{array}{ccc}
1 / \sqrt{2} & 1 / \sqrt{2} & 1 / \sqrt{2} \\
\sin (\omega t) & \sin \left(\omega t-\frac{2 \pi}{3}\right) & \sin \left(\omega t+\frac{2 \pi}{3}\right) \\
\cos (\omega t) & \cos \left(\left(\omega t-\frac{2 \pi}{3}\right)\right. & \cos \left(\omega t+\frac{2 \pi}{3}\right)
\end{array}\right]
$$

(1)

And its inverse transformation matrix can be denoted as $\mathrm{T}^{-1}$ and represented as given in equation 2

$$
\sqrt{2 / 3}\left[\begin{array}{ccc}
1 / \sqrt{2} & \sin (\omega t) & \cos (\omega t) \\
1 / \sqrt{2} & \sin \left(\omega t-\frac{2 \pi}{3}\right) & \cos \left(\left(\omega t-\frac{2 \pi}{3}\right)\right. \\
1 / \sqrt{2} & \sin \left(\omega t+\frac{2 \pi}{3}\right) & \cos \left(\omega t+\frac{2 \pi}{3}\right)
\end{array}\right]
$$

\section{MODIFIED PLL}

A phase locked loop or phase lock loop is a control system that generates output signal whose phase is related to the phase of an input "reference" signal. It is an electronic circuit consisting of a variable frequency oscillator and a phase detector. This circuit compares the phase of the input signal with the phase of the signal derived from its output oscillator and adjusts the frequency of its oscillator to keep the phases matched. The signal from the phase detector is used to control the oscillator in a feedback loop. This paper proposes the usage of Modified PLL[2] as shown in figure 3. A conventional PLL circuit has low performance for highly distorted and unbalanced system voltages. In this paper, the modified PLL is employed for the determination of the positive sequence components of the system voltage signals. The reason behind making a modification in conventional PLL is to improve the UPQC filtering performance under highly distorted and unbalanced voltage conditions.

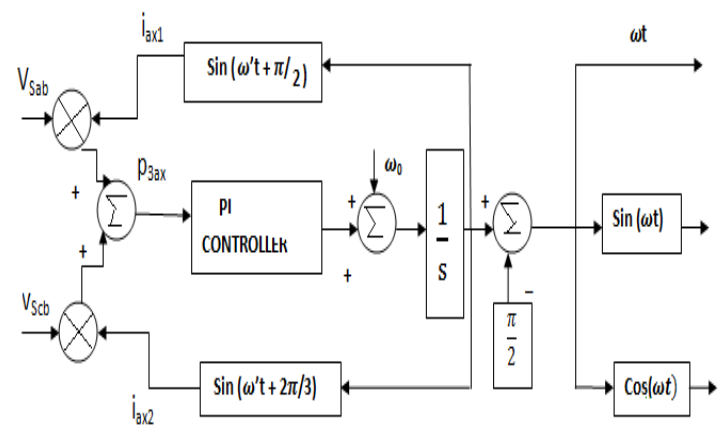

Figure 3: block diagram for Modified PLL

From this figure it is seen that it will get 3 outputs named $\omega t$, which is fed to the transformation matrixes and inverse transformation matrixes to get the corresponding outputs. 


\section{Signal Generation For Shunt Apf}

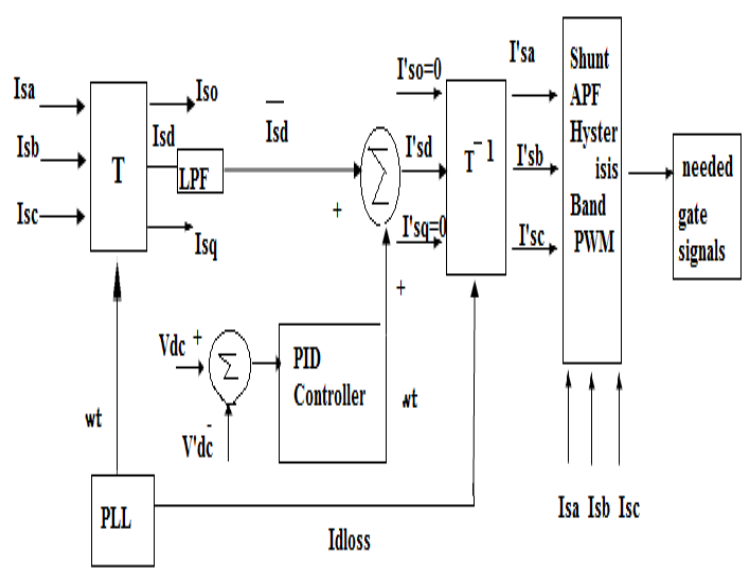

Figure 4: Shunt APF

From the figure 4 the signal generation process of the shunt APF can be understood .Here source currents Isa. Isb, Isc are being given to the transformation matrix and the required output Is0,Isd,Isq is obtained by (1).By passing the positive sequence current(Isd) through an low pass filter $\bar{I} s d$ is obtained .Obtained output is added with the output from the PID controller as follows

$$
\dot{\mathbf{i}}_{\text {sd }}^{\prime}=\dot{\mathbf{1}}_{\text {dloss }}+\overline{\mathbf{1}}_{\text {sd }}
$$

This obtained output is again fed to the inverse transformation matrix alongwith the output obtained from PLL. I'sa,I'sb.I'sc are obtained by (2) respectively .By giving this output to the PWM generator the required gate signals are being obtained which is needed for the perfect working of shunt APF.

\section{PID CONTROLLER}

A Proportional Integral Derivative Controller named as PID controller is a generic control loop feedback mechanism named as controller widely used in industries. A PID controller calculates an "error" value as the difference between a measured process variable and a desired value. The controller attempts to minimize the error by adjusting the process control inputs[8].

The PID controller calculation (algorithm) involves three separate constant parameters, and is accordingly sometimes called three-term control: the proportional, the integral and derivative values, denoted $P, I$, and $D$. Heuristically, these values can be interpreted in terms of time: $P$ depends on the present error, $I$ on the accumulation of past errors, and $D$ is a prediction of future errors, based on current rate of change. The weighted sum of these three actions is used to adjust the process via a control element such as the position of a control valve, a damper, or the power supplied to a heating element.

In the absence of knowledge of the underlying process, a PID controller has historically been considered to be the best controller. By tuning the three parameters in the PID controller algorithm, the controller can provide control action designed for specific process requirements. The working of PID control in this work relies that a comparison is done between the reference dc link voltage and dc link voltage and finally produce Idloss as output, which is finally fed to the inverse transformation matrix for better gating signals. 


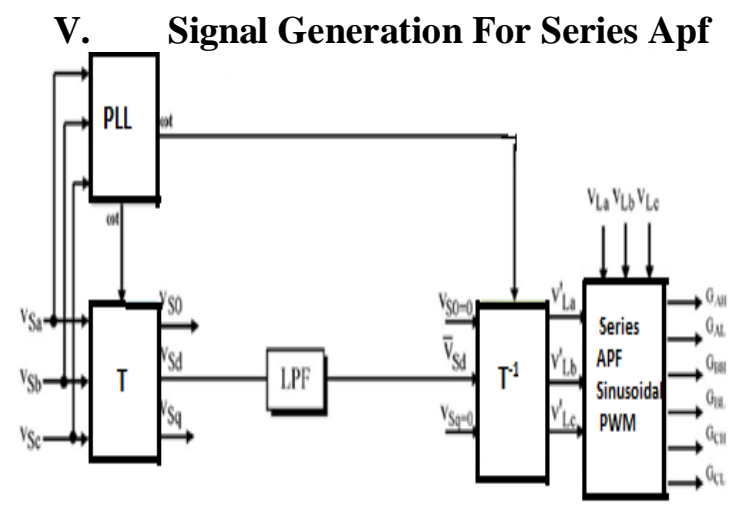

Figure 5:Series APF

In the proposed method shown in figure 5 the series APF controller calculates the reference value to be injected by the STs, comparing the positive-sequence component of the source voltages with load-side line voltages. The algorithm for series APF reference-voltage signal-generation algorithm. The supply voltages Vsabc are transformed to $d-q-0$ by using the transformation matrix $\mathbf{T}$ as given in(1)

The source-voltage positive-sequence average Value, $\bar{V}_{\text {sd }}$ in the $d$-axis is calculated by LPF, as shown in the below equation. Zero and negative sequences of source voltage are set to zero in order to compensate the major power quality problems. The produced load reference voltages and load voltages are compared in the sinusoidal pulse width modulation controller to produce insulated-gate bipolar transistor (IGBT) switching signals and to compensate all voltage-related problems, such as voltage harmonics (2).

\section{Simulation Results}

In this proposed work the main power quality issues namely reactive power compensation, neutral current elimination and harmonics elimination are done.

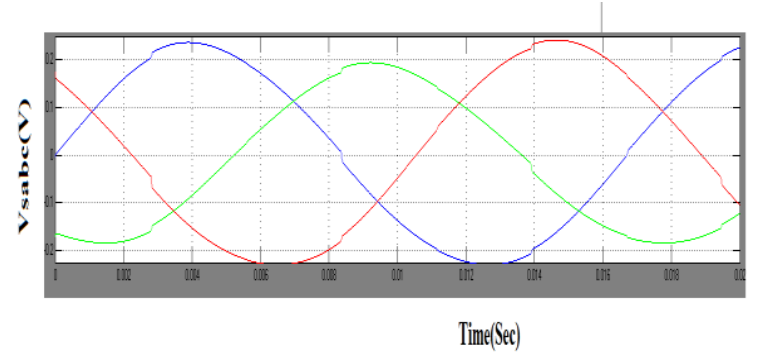

Figure 6.a Unbalanced and distorted main voltage

Output in figure 6.a shows the unbalanced and distorted main voltage which is being fed to the distribution system. The distortions can be viewed from its peak values and the unbalance conditions can be referred from the magnitudes of three phase voltages.

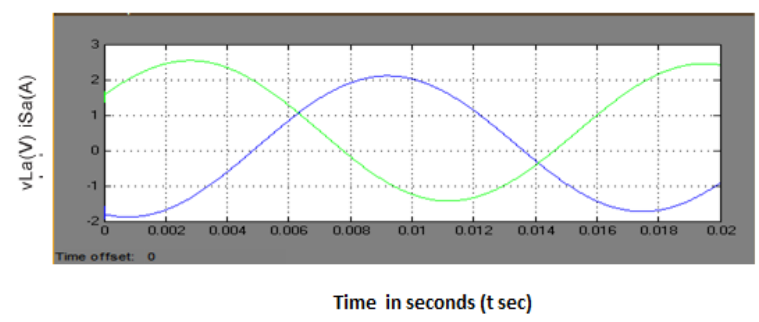

Figure 6.b Reactive Power compensation

In the figure $6 . b$ it shows the reactive compensation has been done .In this figure the voltage leads the current by the application of this effective controlling algorithm and controller .since the controller minimizes the errors that may occur. 


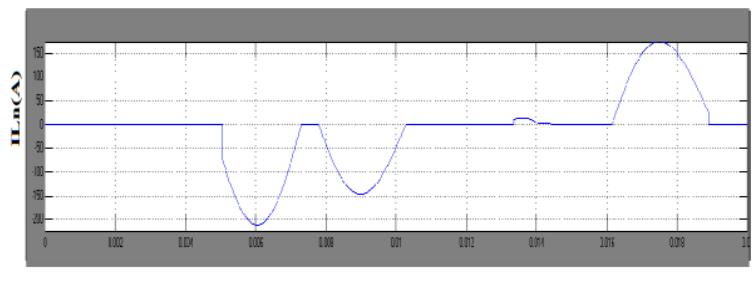

$\mathrm{I}(\mathrm{Sec})$

Figure 6.c Injected compensator current

The figure 6.c shows the injected compensator current, It shows that due to the usage of UPQC an additional must be injected to the shunt APF. By using UPQC with this controlling algorithm a current with maximum compensation has been injected which can be viewed from its varying peaks.

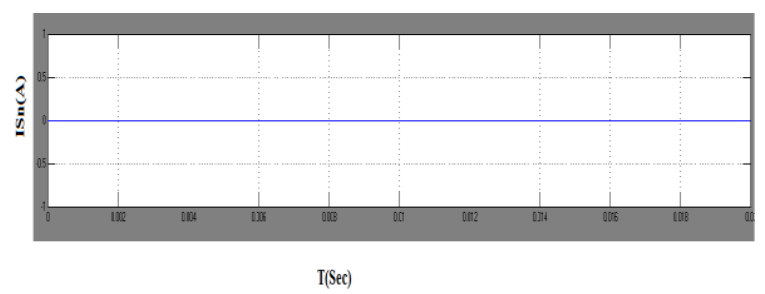

Figure 6.d Source neutral current elimination

Figure 6.d shows the source neutral current, since the source current was fully sinusoidal the neutral current has been reduced approximately to a value of 0amps. This shows the better utilization of SRF theory.

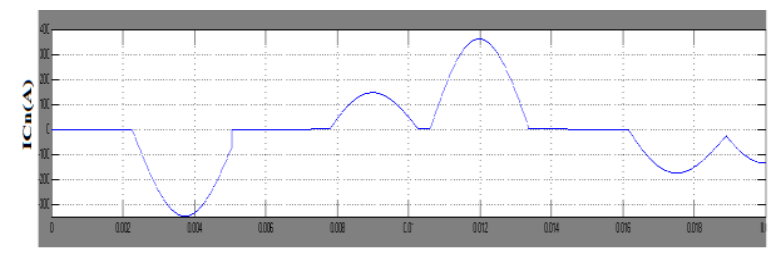

$\mathrm{T}(\mathrm{Sec})$

Figure 6.e Load neutral current elimination

Since the load current was not fully balanced, the neutral current has not been eliminated fully, but it accounts to high percentage reduction as shown in figure 6.e

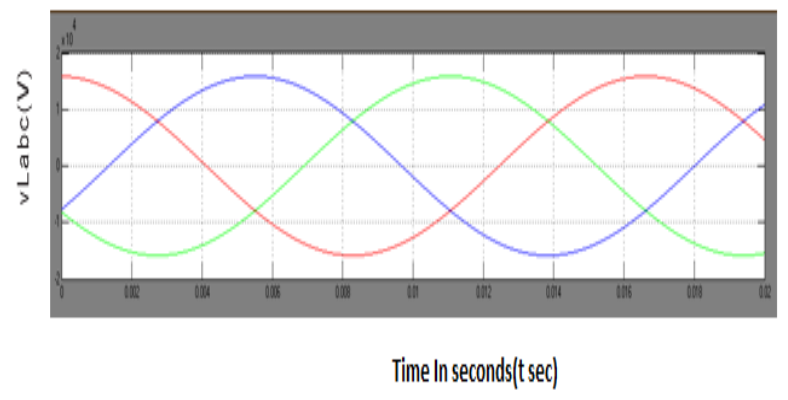

Figure 6.f output voltage waveform

Figure 6.f shows the output voltage waveform. It says that the distortion that occurred at the mains have been completely avoided due to the implementation of UPQC in the system for the compensation.

\section{Conclusions And Future Enhancement}

This paper proposes about Power Quality issues in distribution side and the commonly used device for its effective mitigation. The device named Unified Power Quality Conditioner is being widely used because its 
solely advantage that mitigation of both voltage and current problems by a single device. The performance of this types of devices mainly depends

Various controlling algorithms and the controllers used .In this paper for the effective mitigation of Harmonics Synchronous Reference Frame Controlling algorithm. Since In the synchronously rotating de - qe reference frame, the components at the fundamental frequency, are transformed to dc quantities and all the harmonics are transformed to non-dc quantities and undergo a frequency shift of $60 \mathrm{~Hz}$ in the spectrum. SRF controller extracts the dc quantities by a low-pass filter (LPF) and hence it is insensitive to phase errors. Modified PLL is used in this SRF method due to its low oscillation output. Along with this PID controller has been used in the DC link capacitors for the active power compensation for the series APF. In the future the performance can be investigated by replacing the PID controller used in this paper to Fuzzy controller based on Particle Swarm Optimization algorithm and can find its effectiveness .For a wide research the same can be implemented in Interline UPQC .

\section{References}

[1] Modeling analysis and solution of Power Quality Problems, Mahesh Singh ,Vaibhav Tiwari,

[2] Synchronous reference frame control method for UPQC under unbalanced and distorted load conditions,Metin Kesler and Engin Ozdemir, , ieee transactions on power electronics, vol. 58, sept 2011

[3] Electrical power systems quality, second edition,Roger c.dugan,Surya santaso.

[4] Enhancing Electric Power Quality Using UPQC: A Comprehensive Overview, Vinod Khadkikar, ieee transactions on power electronics, vol. 27, may 2012

[5] Performance Analysis of Various SRF Methods in Three Phase Shunt Active Filters, Naimish Zaveri,ICIIS 2009 , 28 - 31 December 2009, Sri Lanka

[6] Operation of a phase locked loop system under distorted utility conditions, Vikrarn Kaura, Vladimir Blasko,1996

[7] Power System State Estimation and Contingency Constrained Optimal Power Flow - A Numerically Robust Implementation, Slobodan Paji, April 2007

[8] Probably the best simple PID tuning rules in the world. Sigurd Skogestad, September 12, 2001 